\title{
Fresnel Diffraction from Polygonal Apertures
}

\author{
Jungang Huang, J. M. Christian and G.S. McDonald \\ Joule Physics Laboratory, School of Computing, Science and Engineering, Institute of Materials Research, \\ University of Salford, Salford M5 4WT, U.K.
}

\section{Introduction}

We present, for the first time, two complementary analytical techniques for calculating the Fresnel diffraction patterns from a polygonal aperture illuminated by a plane wave. These frameworks are exact, in that they do not involve any further approximation beyond the (paraxial) Fresnel integral. Here, we consider regular polygonal apertures, but our results are readily extended to describe near-field diffraction from closed apertures of arbitrary shape. Our results are of fundamental importance and have specific applications where standard methods, such as Fast Fourier Transform (FFT) techniques, fail. For example, in unstable-resonator mode calculations, both (paraxial beam) ABCD matrix modelling and existing semi-analytical methods can only give accurate results in limited parameter regimes. Consequently, a complete and detailed study of optical fractal laser modes [1] has not previously been possible. A specific advantage of our formulisms is the ability to calculate and store the fine details of only a small portion of one, or many, complex diffraction patterns.

Moreover, the explicit mathematical form of our results may also lend physical insight into a wide range of diffraction-related phenomena in physics. For example, insight into the physical origin of excess quantum noise in lasers, where the transverse symmetry of an aperturing element has been shown to play a central role in the observed phenomena [2]. While Fraunhoffer (far-field) diffraction patterns have been known for many years, there has been relatively little equivalent published work in the Fresnel (near-field) regime. The far-field approximation allows the expression of diffraction patterns and descriptions of derivative concepts (eg in holography, filtering, convolution and coherence) as simple Fourier integrals and transform theorems, respectively. Our new results permit the mathematical and physical expression of near-field diffraction patterns in terms of their elemental spatial structures (edge-waves). It is plausible that our results may also open future doors in the development of derivative concepts in Fresnel Optics.

\section{Analytical Descriptions and Results}

The Fresnel diffraction patterns consist of a plane-wave (uniform) component, plus a rapidly-varying interference contribution from boundary-diffracted waves from all edges of the aperture. This physical interpretation is present in the mathematical formulations of both the S-Function Method, which deals explicitly with edge-wave combinations, and also the Line-Integral Method, where the Fresnel integral over the aperture area is expressed as a circulation around its edge. Extensive computational investigations have verified that our two approaches are completely equivalent, and produce identical results. The amount of fine detail present in the pattern can be quantified by the Fresnel Number $N=a^{2} / \lambda L+f(n)$, where $a$ is the radius of a circle inscribing an $n$-sided polygon, $L$ is the distance from the aperture to the observation plane $\left(L^{2} \gg a^{2}\right.$ is a paraxial approximation inherent to the near-field approximation), $\lambda$ is the wavelength of the illuminating light, and $f(n)=0.30618 n^{2}-0.19533 n-0.68095$ is a term allowing for the geometrical structure of the aperture. As $n$ becomes larger, the level of detail increases (see Fig. 1).
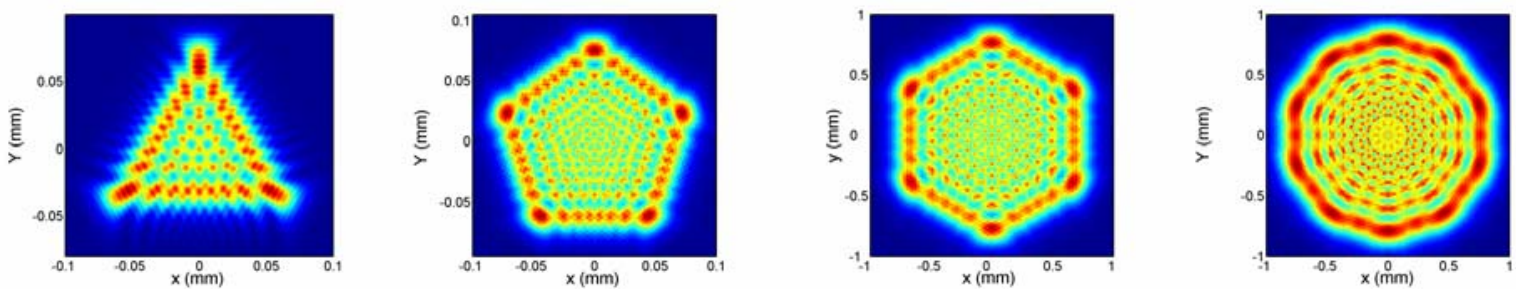

Fig. 1. Diffraction patterns for (left to right) triangle-, pentagon-, hexagon-, and decahedron-shaped apertures. Parameters are $\lambda=0.5 \mu \mathrm{m}, a=1 \mathrm{~mm}, L=100 a$. In these cases, $n=3,5,6$ and 10 , respectively.

\section{References}

[1] G.P. Karman, G.S. McDonald, G.H.C. New, and J.P. Woerdman, Nature 402 (1999) 138.

[2] G.P. Karman, G.S. McDonald, J.P. Woerdman, and G.H.C. New, Appl Opt 38 (1999) 6874. 